\title{
OPEN The limit of tolerable micromotion for implant osseointegration: a systematic review
}

\begin{abstract}
Nupur Kohli, Jennifer C. Stoddart \& Richard J. van Arkel ${ }^{\bowtie}$
Much research effort is being invested into the development of porous biomaterials that enhance implant osseointegration. Large micromotions at the bone-implant interface impair this osseointegration process, resulting in fibrous capsule formation and implant loosening. This systematic review compiled all the in vivo evidence available to establish if there is a universal limit of tolerable micromotion for implant osseointegration. The protocol was registered with the International Prospective Register for Systematic Reviews (ID: CRD42020196686). Pubmed, Scopus and Web of Knowledge databases were searched for studies containing terms relating to micromotion and osseointegration. The mean value of micromotion for implants that osseointegrated was $32 \%$ of the mean value for those that did not $(112 \pm 176 \mu \mathrm{m}$ versus $349 \pm 231 \mu \mathrm{m}, p<0.001)$. However, there was a large overlap in the data ranges with no universal limit apparent. Rather, many factors were found to combine to affect the overall outcome including loading time, the type of implant and the material being used. The tables provided in this review summarise these factors and will aid investigators in identifying the most relevant micromotion values for their biomaterial and implant development research.
\end{abstract}

Metallic protheses implanted directly into bone have revolutionised the treatment of dental, orthopaedic and spinal disease, pain and trauma, with millions of procedures performed annually worldwide ${ }^{1}$. This has resulted in a continued drive from research centres of excellence and industry to develop new technology that improves outcomes, reduces revision rates, and enables treatment for more patients' groups, such as those that are younger and more active.

In silico and in vitro modelling are at the heart of the pre-clinical development process for new implant technologies. In the field of implant fixation, a common parameter investigated is the amount of oscillatory micromotion at the bone-implant interface ${ }^{2-4}$. Micromotion is the temporary localised relative movement that occurs between an implant surface and adjacent bone when functional loading is applied ${ }^{5,6}$; with any permanent displacement known as subsidence/migration. These sub-millimetre (hence micro) motions are too small to be seen by the naked eye. Micromotion is the result of primary implant instability and differing bone/implant material moduli, and consequently depends on the implant material, bone density, implant/bone geometry, surgical technique, and the level of interference fit, as well as the magnitude and direction of the applied loading ${ }^{4-9}$.

Micromotion is investigated as in vivo data suggest that too much of it leads to failed implant osseointegration: a fibrous capsule forms around the implant rather than a direct structural and functional connection between the host bone and implant ${ }^{10,11}$. Failed osseointegration leads to aseptic implant loosening, implant failure and the need for expensive revision surgery, both financially and in terms of quality of life ${ }^{12}$. Thus, much research time and resource has been invested to ensure new implant designs and surgical techniques result in acceptable/ improved micromotion at the bone-implant interface ${ }^{13-22}$. There even exists an ASTM standard (F2537-06) to ensure micromotion is measured accurately and repeatably.

Micromotion data are often compared to a limit, below which the implant is considered to pass/be suitable for clinical use, and above which it is considered to be at risk of failed osseointegration. Early in vivo research suggested an upper limit of $150 \mu \mathrm{m}$ of micromotion and over time this value has become an oft-cited gold standard ${ }^{3,23-28}$. However, the evidence in the 1990s for the $150 \mu \mathrm{m}$ limit was inconclusive and much time has subsequently elapsed meaning that there is likely much more data available with which to draw conclusions about the relationship between micromotion and osseointegration ${ }^{29-31}$.

This systematic review aimed to compile all the quantitative in vivo evidence relating micromotion to osseointegration to answer the following research questions: (1) Is there value of micromotion that can be universally 


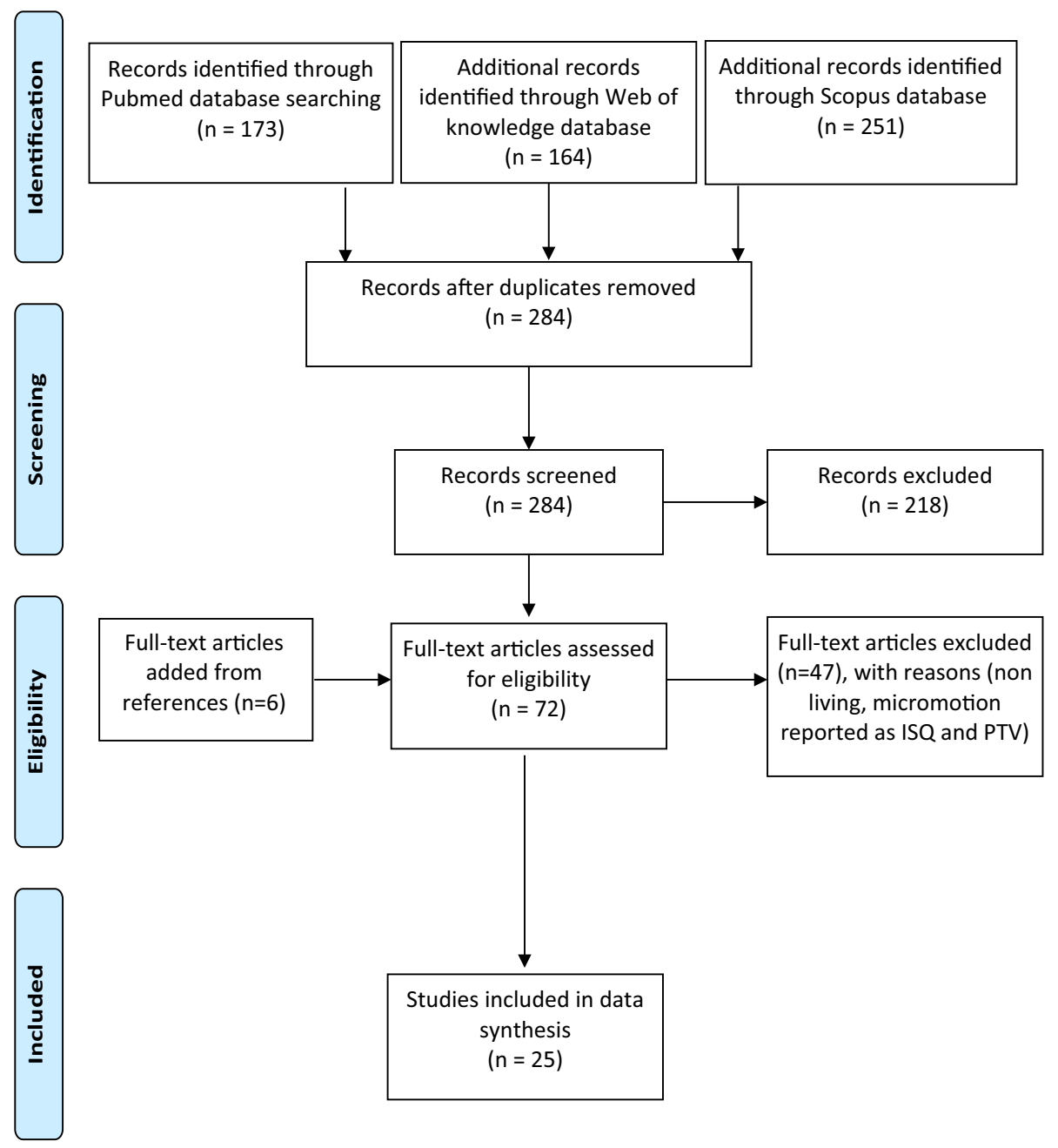

Figure 1. Flowchart of the study selection process.

used as a limit for in vitro or in silico modelling? (2) To what extent is micromotion correlated with bone-implant contact? (3) Which factors influence the relationship between micromotion and osseointegration?

\section{Results}

Study selection and characteristics. 284 unique records were identified from the databases (Fig. 1). After initial screening, 218 articles were excluded leaving 66 studies for full article screening. An additional 6 studies that passed all inclusion criteria were identified from these 66 studies. After full-text screening, 25 studies were found to be eligible for the quantitative analysis (Table 1).

Micromotion and osseointegration. One human and twenty-four animal studies were identified (Table 1). For the human post-mortem study, the micromotion for osseointegrated hip stems was less than $40 \mu \mathrm{m}$ which compared to $150 \mu \mathrm{m}$ for a stem with failed bone ingrowth. For the animal studies, the mean value of micromotion for implants that osseointegrated was $32 \%$ of the mean value for those that did not $(112 \pm 176 \mu \mathrm{m}$ osseointegrated versus $349 \pm 231 \mu \mathrm{m}$ non-osseointegrated, Mann Whitney test $p<0.001$, Fig. 2 ). However, the osseointegration outcome also depended on other experimental/implant conditions with no distinct osseointegration limit detected. Rather, the range for successful/failed osseointegration overlapped: 15 to $750 \mu \mathrm{m}$ for osseointegrated samples versus 30 to $750 \mu \mathrm{m}$ for non-osseointegrated samples (Table 1 and Fig. 2).

The effects of research method: applied vs measured micromotion. When micromotion was applied, lower micromotion resulted in more consistent osseointegration (Fig. 3A, Mann Whitney $p$ value $=0.001$ ). Similarly, when micromotion was measured at the end of the study duration, implants that osseointegrated had lower micromotion than implants that did not (Fig. 3B, Mann Whitney $p$ value $=0.01$ ). Comparing values of micromotion between the methods (measured vs applied), no differences were observed for the osseointegrated group, and similarly there was no difference between the methods for the non-osseointegrated group. (Fig. 3). 


\begin{tabular}{|c|c|c|c|c|c|c|}
\hline Author & Year & Country & Micromotion OI $(\mu \mathrm{m})$ & $\begin{array}{l}\text { Micromotion Non-OI } \\
(\mu \mathrm{m})\end{array}$ & Applied or measured & Animal or Human \\
\hline Aspenberg $^{32}$ & 1992 & Sweden & N/A & 500 & \multirow{18}{*}{ Applied } & \multirow{25}{*}{ Animal } \\
\hline Bragdon $^{33}$ & 1996 & USA & 20 & 40,150 & & \\
\hline Duyck $^{34}$ & 2006 & Belgium & 60 & 30,90 & & \\
\hline Goodman $\mathrm{a}^{35}$ & 1995 & USA & N/A & 500,500 & & \\
\hline Goodman $b^{31}$ & 1993 & Sweden & 750 & 750 & & \\
\hline Goodman $\mathrm{c}^{36}$ & 1994 & Sweden & $500^{*} 0$ & 500 & & \\
\hline Goodman $\mathrm{d}^{37}$ & 1993 & Sweden & 500 & 500 & & \\
\hline Jakobsen a $^{38}$ & 2015 & Denmark & N/A & 500 & & \\
\hline Jakobsen b ${ }^{39}$ & 2017 & Denmark & N/A & 500,500 & & \\
\hline Jasty $^{40}$ & 1997 & USA & 20 & 40,150 & & \\
\hline Kawahara $^{29}$ & 2003 & Japan & 30 & 580,630 & & \\
\hline Overgaard $^{41}$ & 1996 & Denmark & $150^{*} 0$ & $150 * 12$ & & \\
\hline Soballe $\mathrm{a}^{42}$ & 1992 & Denmark & N/A & 500,500 & & \\
\hline Soballe $b^{43}$ & 1992 & Denmark & 150 & 150 & & \\
\hline Vandamme $\mathrm{a}^{30}$ & 2007 & Belgium & 30,50 & 30 & & \\
\hline Vandamme $\mathrm{b}^{44}$ & 2007 & Belgium & 30,90 & N/A & & \\
\hline Vandamme $\mathrm{c}^{45}$ & 2008 & Belgium & 30,30 & N/A & & \\
\hline Vandamme $\mathrm{d}^{46}$ & 2007 & Belgium & 30,30 & N/A & & \\
\hline Manley $^{25}$ & 1995 & USA & $33 \pm 23.7,17 \pm 4.2$ & N/A & \multirow{7}{*}{ Measured } & \\
\hline Pilliar $^{47}$ & 1986 & Canada & 28 & 150 & & \\
\hline Trisi a ${ }^{48}$ & 2017 & Italy & $77.9 \pm 17.29,75.3 \pm 19$ & N/A & & \\
\hline \multirow{2}{*}{ Trisi b $b^{8}$} & \multirow{2}{*}{2015} & \multirow{2}{*}{ Italy } & $64 \pm 27$ & $177 \pm 87$ & & \\
\hline & & & $15 \pm 5,22 \pm 6$ & N/A & & \\
\hline Trisi $\mathrm{c}^{49}$ & 2016 & Italy & $\begin{array}{l}94.88 \pm 10.94 \\
60.45 \pm 5.29\end{array}$ & N/A & & \\
\hline Trisi d ${ }^{50}$ & 2016 & Italy & $161.26 \pm 134.39$ & $619.5 \pm 328.26$ & & \\
\hline Engh $^{51}$ & 1992 & USA & $<40$ & 150 & Measured & Human \\
\hline
\end{tabular}

Table 1. Osseointegrated (OI) and non-osseointegrated (Non-OI) values of micromotion ( $\mu \mathrm{m})$ from the studies selected For applied values, the value was set as a controlled experimental parameter, for measured values means and standard deviation are reported where possible. ${ }^{\star} 0$ represents experiments with immobilized implants after a period of loading. ${ }^{\star} 12$ represents experiments that applied an additional implant displacement for 12 weeks.

Micromotion and bone-implant-contact. Out of the 24 animal studies, 13 studies examined osseointegration as the percentage of BIC, 2 studies reported on bone ingrowth and 1 study reported on both BIC and bone ingrowth. For implants that were defined as osseointegrated, a positive correlation was observed between micromotion and \% BIC (Spearman's $\rho=0.41, p$ value $=0.02$ ). Micromotion and BIC were not correlated for the non-osseointegrated group $(p$ value $=0.39)$, nor the full dataset $(p$ value $=0.07)$.

Observation time and Bone-implant-contact. There was a positive correlation between observation time and percentage BIC, with longer study duration time resulting in better percentage BIC (Spearman's $\rho=0.40, p$ value $=0.01)$.

\section{Discussion}

The most important finding of this systematic review was that the available data refutes the idea of a universal limit of tolerable micromotion for implant osseointegration. Whilst on average, the micromotion associated with osseointegration was $32 \%$ of the micromotion associated with failed fixation, many exceptions to the rule were identified (Fig. 2). In some studies, micromotion at the bone-implant interface as high as $750 \mu \mathrm{m}$ osseointegrated, whilst in other micromotion as low as $30 \mu \mathrm{m}$ did not osseointegrate. Thus, implant and external factors must be considered when estimating the level of micromotion that could lead to successful osseointegration for a new biomaterial/implant. The following implant factors were associated with higher levels of micromotion and successful osseointegration: hydroxyapatite coating ${ }^{43}$, larger threads in lower density bone ${ }^{8}$, and square pore cross-sectional shape ${ }^{37}$. The following environmental factors were associated with higher levels of micromotion and successful osseointegration: infrequent loading ${ }^{31}$, a rest period following initial loading ${ }^{41}$ and longer study duration (9 weeks or more) ${ }^{30,44}$ (Table 2).

The gold standard micromotion limit is often considered $150 \mu \mathrm{m}$. However, Overgaard et al., and Soballe et al., showed that this level of micromotion can be tolerated if a period of rest is allowed after initial loading and if the implant was coated with hydroxyapatite ${ }^{41,43}$. The accelerated resorption of HA coating under excessive micromotion could have led to a better bony ingrowth as studies have previously shown that HA coating on 
A

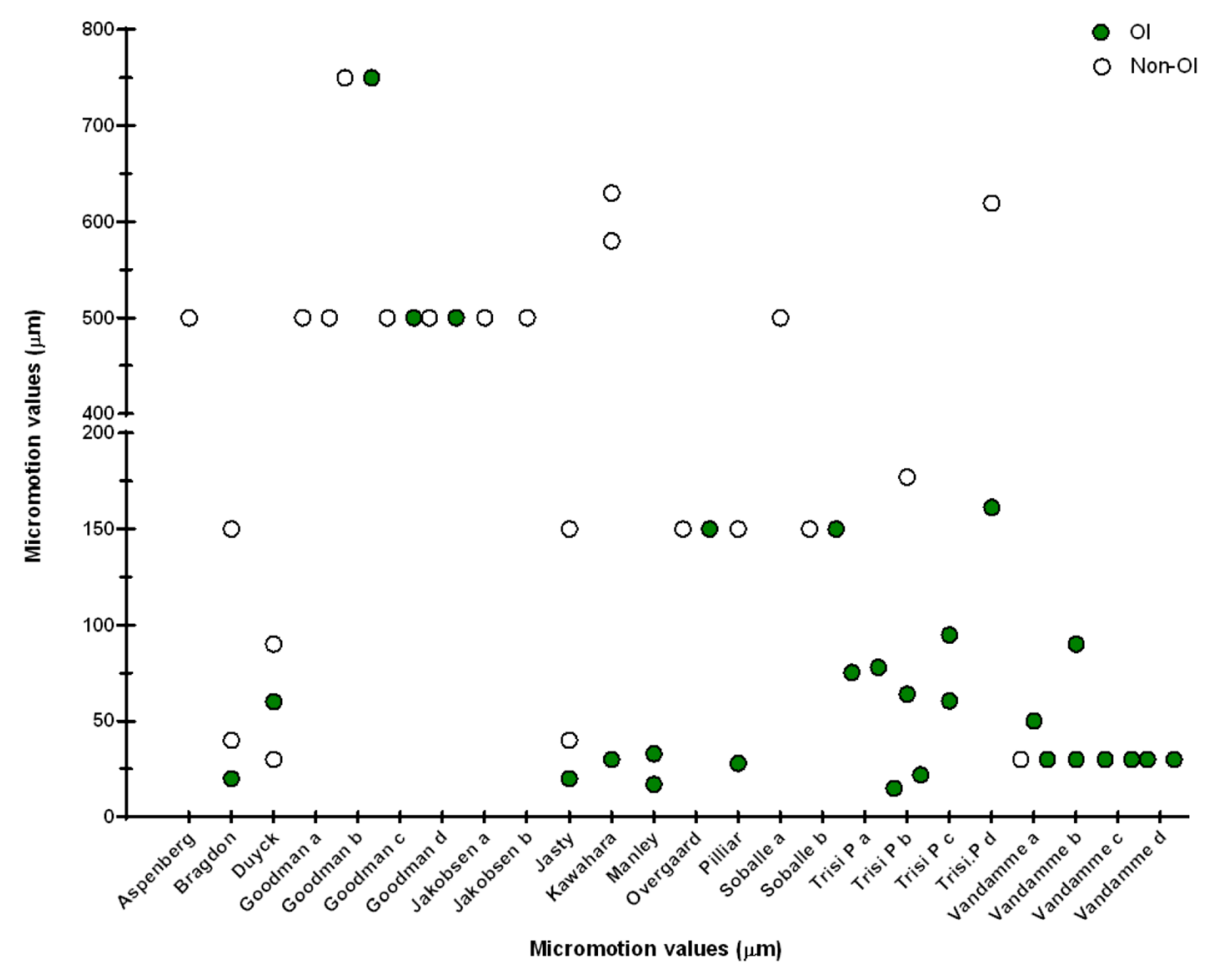

B

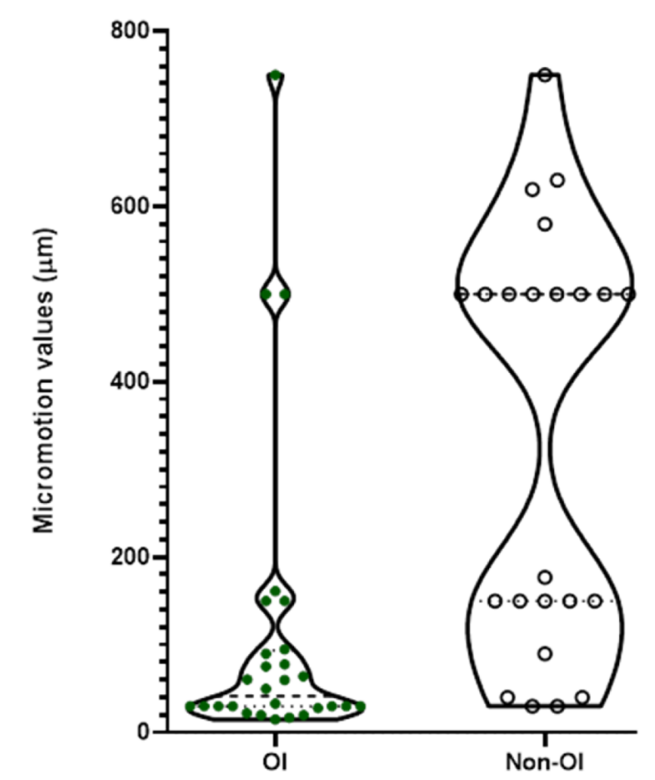

Figure 2. (A) Scatterplot of the animal data showing the micromotion value for osseointegrated (green, circle, $n=28$ ) and non-osseointegrated (hollow circles, $n=23$ ) samples. (B) Violin plot of the same data. Whilst micromotion was lower for the osseointegrated samples (Mann Whitney test $p<0.001$ ), there was also considerable overlap between the groups.

titanium implants improve BIC through its direct interaction with osteoblast, osteoclasts and pro-inflammatory markers ${ }^{52,53}$. Further, previous studies have also shown that a period of rest or otherwise referred to as recovery phase may be beneficial for BIC. The recovery phase or time off helps to counteract the waning effects of long-term mechanical loading, and improve the responsiveness of osteoblasts and osteocytes to restart bone formation ${ }^{54,55}$. Goodman et al., showed that oscillatory motions up to $750 \mu \mathrm{m}$ once a day would allow successful osseointegration, while the same motions twice a day would not ${ }^{31}$, emphasizing the effect of loading duration. Similarly, Goodman also demonstrated how implant factors can influence the tolerable micromotion: by changing 
A

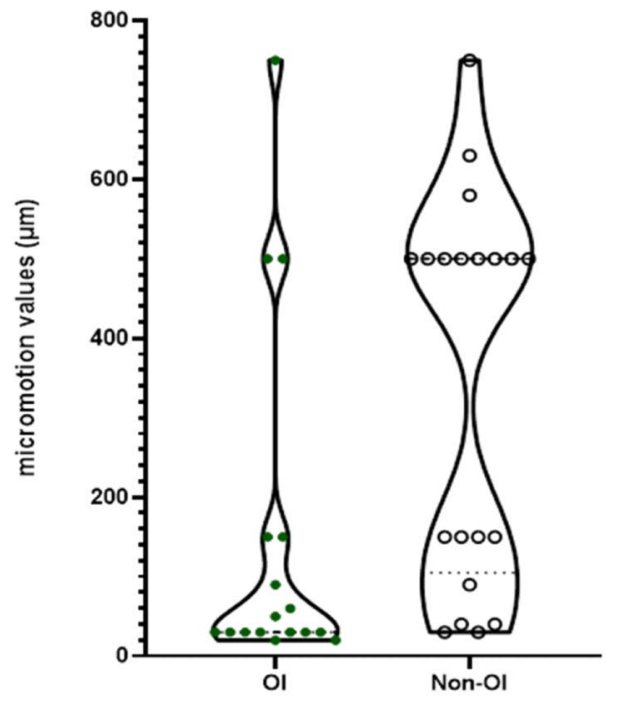

B

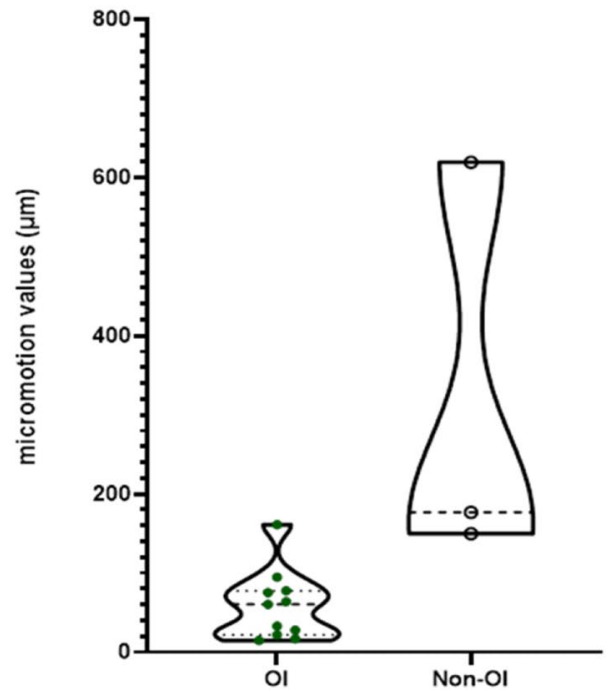

Figure 3. (A) Applied values of micromotion in osseointegrated (OI, $\mathrm{n}=17)$ and non-osseointegrated (Non-OI, $\mathrm{n}=20$ ) groups for the animal studies. Mann Whitney $p$ value $=0.001^{\star *}$. (B) Measured values of micromotion in OI $(n=11)$ and non-OI $(n=3)$ for the animal studies. Mann Whitney $p$ value $=0.003 * *$.

the pore-cross sectional shape of the outer bone chamber from round to square in the traditional bone chamber designs, bony ingrowth would be facilitated even with micromotions as high as $500 \mu \mathrm{m}$.

Whilst the data reviewed revealed that the mean value of micromotion for successful osseointegration was $112 \mu \mathrm{m}$, some studies showed that micromotion as low as $30 \mu \mathrm{m}$ can lead to failed implant fixation ${ }^{30,40}$. The authors attributed this to the duration of loading, hypothesizing that the process of bone formation had not been reached within 6 weeks. Subsequent experiments measured osseointegration at 9 weeks or more and demonstrated successful osseointegration with the longer study duration ${ }^{30,44}$. The data from this systematic review further supports this finding, demonstrating a positive correlation between osseointegration (measured by percentage BIC) and study duration (Fig. 4). Biologically, osseointegration starts with woven bone formation, followed a period of remodelling to lamellar bone in response to mechanical loading. This transition from woven bone to lamellar bone formation takes starts around 6-8 weeks and can take a period of months to complete ${ }^{56,57}$. Therefore, in vivo experimental studies exploring osseointegration of implants should allow a time period of over 6 weeks to see the full healing response. The effects of study duration have also been reflected by recent computational research which highlighted the differing mechanisms between bone healing and remodelling, and hence the importance of the measurement time point ${ }^{2}$.

Another explanation for the contradictory in vivo data is that micromotion is a simplified, clinically convenient measure, which overlooks the fundamental mechanobiological mechanisms that drive implant osseointegration. In vivo data coupled with finite element analyses suggest that it is the interfacial stress-strain state resulting from implant micromotion that stimulates osseointegration ${ }^{58,59}$. Different loading conditions (axial, shear, torsional, etc.), combined with different localised implant/bone geometry lead to different stress-strain states, with too much strain leading to fibrous tissue formation ${ }^{58-61}$. Indeed, by considering the interfacial stress-strain state, it is possible to relate implant bony ingrowth theory $y^{2,58,59}$ to fracture healing theory ${ }^{62-65}$, which intuitively one would expect given the involvement of the same cell types ${ }^{66}$. Conversely, when the implant/environmental conditions that affect the interfacial stress-strain state are ignored, counter-intuitive trends can be observed. For example, when neglecting these factors, it was found that increased micromotion was positively correlated with increased percentage BIC (Fig. 5). However, within study data demonstrate that micromotion and percentage $\mathrm{BIC}$ are negatively correlated ${ }^{50}$. This further emphasises the needs to consider implant and environmental factors and their link to the interfacial stress-strain state when interpreting how micromotion affects osseointegration. It should also be noted that there is no standard interpretation of BIC and so caution should also be applied when interpreting BIC between studies. Some studies report BIC as the fraction of mineralized bone in direct contact with the implant surface ${ }^{44}$, whilst other describe it as the length of the implant surface in contact with (both mineralised and non-mineralised) bone relative to the total implant length ${ }^{34}$.

Historically, the causal effect of implant micromotion on osseointegration was investigated by applying known displacement and subsequently measuring osseointegration. However, more recently, research method has shifted to applying known loads and quantifying micromotion at the end of the experiment ${ }^{4,8}$. Both measurement techniques were able to identify differences in micromotion between implants that osseointegrated and those that did not. Interestingly, when comparing the results between the two different methods, no differences were found.

The majority of studies identified applied micromotion as a controlled experimental condition, meaning that mean and standard deviation micromotion data were not available prohibiting application of the established meta-analysis approaches recommended by Borenstein et $a^{67}$. For the same reason, it was not possible to perform 


\begin{tabular}{|c|c|c|c|c|c|c|c|c|c|c|c|c|c|c|c|}
\hline \multirow[b]{2}{*}{ Author } & \multirow[b]{2}{*}{ Year } & \multirow[b]{2}{*}{$\begin{array}{l}\text { No. of } \\
\text { animals or } \\
\text { patients }\end{array}$} & \multirow[b]{2}{*}{ Species } & \multirow{2}{*}{$\begin{array}{l}\text { No. of } \\
\text { samples } \\
\text { (per } \\
\text { group) }\end{array}$} & \multirow[b]{2}{*}{$\begin{array}{l}\text { No. of } \\
\text { study } \\
\text { groups }\end{array}$} & \multirow[b]{2}{*}{$\begin{array}{l}\text { Implant } \\
\text { material }\end{array}$} & \multirow[b]{2}{*}{$\begin{array}{l}\text { Implant } \\
\text { coating or } \\
\text { implant type }\end{array}$} & \multirow[b]{2}{*}{ Bone } & \multirow[b]{2}{*}{\begin{tabular}{|l} 
Time \\
(weeks)
\end{tabular}} & \multirow[b]{2}{*}{$\begin{array}{l}\text { Loading } \\
\text { conditions }\end{array}$} & \multirow[b]{2}{*}{$\begin{array}{l}\text { Loading } \\
\text { cycles and } \\
\text { time }\end{array}$} & \multicolumn{2}{|c|}{ Micromotion $(\mu \mathrm{m})$} & \multirow[b]{2}{*}{$\begin{array}{l}\text { Bone } \\
\text { ingrowth }\end{array}$} & \multirow[b]{2}{*}{$\begin{array}{l}\text { Bone- } \\
\text { implant- } \\
\text { contact }\end{array}$} \\
\hline & & & & & & & & & & & & $\begin{array}{l}\text { Osseo- } \\
\text { integrated }\end{array}$ & $\begin{array}{l}\text { Non-osseo- } \\
\text { integrated }\end{array}$ & & \\
\hline \multirow[t]{2}{*}{$\begin{array}{l}\text { Aspen- } \\
\text { berg }\end{array}$} & 1992 & 6 & rabbits & 13 & 2 & titanium & none & Tibia & 3 & Unloaded & $\mathrm{N} / \mathrm{A}$ & Unloaded & & & Not measured \\
\hline & & & & 15 & & & & & 3 & $\begin{array}{l}500 \mu \mathrm{m} \text { Micro- } \\
\text { motion }\end{array}$ & $\begin{array}{l}20 \text { cycles/ } \\
\text { day }\end{array}$ & & 500 & & \\
\hline \multirow[t]{4}{*}{ Bragdon } & 1996 & 20 & Dogs & 5 & 4 & Titanium & None & Femur & 6 & UNLOADED & N/A & Unloaded & & & Not measured \\
\hline & & & & & & & & & 6 & $\begin{array}{l}20 \mu \mathrm{m} \text { Micro- } \\
\text { motion }\end{array}$ & $8 \mathrm{~h} /$ day & 20 & & & \\
\hline & & & & & & & & & 6 & $\begin{array}{l}40 \mu \mathrm{m} \text { micro- } \\
\text { motion }\end{array}$ & $8 \mathrm{~h} /$ day & & 40 & & \\
\hline & & & & & & & & & 6 & $\begin{array}{l}150 \mu \mathrm{m} \text { micro- } \\
\text { motion }\end{array}$ & $8 \mathrm{~h} /$ day & & 150 & & \\
\hline \multirow[t]{4}{*}{ Duyck } & 2006 & 10 & Rabbits & 10 & 4 & Titanium & None & Tibia & 6 & Unloaded & & Unloaded & & & $20-25 \%$ \\
\hline & & & & & & & & & 6 & $\begin{array}{l}30 \mu \mathrm{m} \text { micro- } \\
\text { motion }\end{array}$ & $\begin{array}{l}800 \text { cycles/ } \\
\text { day; twice/ } \\
\text { week }\end{array}$ & & 30 & & $5-10 \%$ \\
\hline & & & & & & & & & 6 & $\begin{array}{l}60 \mu \mathrm{m} \text { micro- } \\
\text { motion }\end{array}$ & $\begin{array}{l}800 \text { cycles/ } \\
\text { day; twice/ } \\
\text { week }\end{array}$ & 60 & & & $15-20 \%$ \\
\hline & & & & & & & & & 6 & $\begin{array}{l}90 \mu \mathrm{m} \text { micro- } \\
\text { motion }\end{array}$ & $\begin{array}{l}800 \text { cycles/ } \\
\text { day; twice/ } \\
\text { week }\end{array}$ & & 90 & & $5-10 \%$ \\
\hline \multirow[t]{4}{*}{$\begin{array}{l}\text { Good- } \\
\text { man a }\end{array}$} & 1995 & 9 & Rabbits & 9 & 4 & Titanium & $\begin{array}{l}\text { Micromotion } \\
\text { alone }\end{array}$ & Femur & 3 & $\begin{array}{l}500 \mu \mathrm{m} \text { micro- } \\
\text { motion }\end{array}$ & $\begin{array}{l}40 \text { cycles/ } \\
\text { day }\end{array}$ & & 500 & $25 \pm 6$ & \\
\hline & & & & & & & $\begin{array}{l}\text { Polyethylene } \\
\text { particles only }\end{array}$ & & 3 & Unloaded & N/A & & Unloaded & $23 \pm 9$ & \\
\hline & & & & & & & $\begin{array}{l}\text { No polyeth- } \\
\text { ylene }\end{array}$ & & 3 & Unloaded & N/A & Unloaded & & $33 \pm 6$ & \\
\hline & & & & & & & $\begin{array}{l}\text { polyethyl- } \\
\text { ene+micro- } \\
\text { motion }\end{array}$ & & 3 & $\begin{array}{l}500 \mu \mathrm{m} \text { micro- } \\
\text { motion }\end{array}$ & $\begin{array}{l}40 \text { cycles/ } \\
\text { day }\end{array}$ & & 500 & $23 \pm 9$ & \\
\hline \multirow[t]{3}{*}{$\begin{array}{l}\text { Good- } \\
\text { man b }\end{array}$} & 1993 & 7 & Rabbits & 7 & 3 & Titanium & None & & 3 & Unloaded & & Unloaded & & & $31 \pm 2 \%$ \\
\hline & & & & 10 & & & & Tibia & 3 & $\begin{array}{l}750 \mu \mathrm{m} \text { micro- } \\
\text { motion }\end{array}$ & $\begin{array}{l}20 \text { cycles/ } \\
\text { day }\end{array}$ & 750 & & & $46 \pm 5 \%$ \\
\hline & & & & 7 & & & & & 3 & $\begin{array}{l}750 \mu \mathrm{m} \text { micro- } \\
\text { motion }\end{array}$ & $\begin{array}{l}20 \text { cycles } \\
\text { twice/day }\end{array}$ & & 750 & & $19 \pm 7 \%$ \\
\hline \multirow[t]{3}{*}{$\begin{array}{l}\text { Good- } \\
\text { man c }\end{array}$} & 1994 & 5 & Rabbits & 5 & 3 & Titanium & None & Tibia & 6 & $\begin{array}{l}500 \mu \mathrm{m} \\
\text { micromotion } \\
\text { (3 weeks), } \\
\text { then unloaded } \\
\text { ( } 3 \text { weeks) }\end{array}$ & $\begin{array}{l}40 \text { cycles/ } \\
\text { day then } \\
\text { unloaded }\end{array}$ & 500 & & & $37 \pm 7$ \\
\hline & & & & & & & & & 3 & $\begin{array}{l}500 \mu \mathrm{m} \text { micro- } \\
\text { motion }\end{array}$ & & & 500 & & $20 \pm 2$ \\
\hline & & & & & & & & & 3 & Unloaded & N/A & unloaded & & & $37 \pm 6$ \\
\hline \multirow[t]{2}{*}{$\begin{array}{l}\text { Good- } \\
\text { man d }\end{array}$} & 1993 & 10 & Rabbits & 6 & 2 & Titanium & $\begin{array}{l}\text { Square } \\
\text { chamber }\end{array}$ & Tibia & 3 & $\begin{array}{l}500 \mu \mathrm{m} \text { micro- } \\
\text { motion }\end{array}$ & $\begin{array}{l}20 \text { cycles/ } \\
\text { day }\end{array}$ & 500 & & & not measured \\
\hline & & & & 5 & & & \begin{tabular}{|l|}
$\begin{array}{l}\text { Round } \\
\text { chamber }\end{array}$ \\
\end{tabular} & & 3 & $\begin{array}{l}500 \mu \mathrm{m} \text { micro- } \\
\text { motion }\end{array}$ & $\begin{array}{l}20 \text { cycles/ } \\
\text { day }\end{array}$ & & 500 & & not measured \\
\hline $\begin{array}{l}\text { Jakob- } \\
\text { sen a }\end{array}$ & 2015 & 10 & Sheep & 10 & 2 & PMMA & & Femur & 12 & $\begin{array}{l}500 \mu \mathrm{m} \text { micro- } \\
\text { motion }\end{array}$ & $\begin{array}{l}\text { Every gait } \\
\text { cycle }\end{array}$ & Unloaded & 500 & & \\
\hline \multirow[t]{2}{*}{$\begin{array}{l}\text { Jakob- } \\
\text { sen b }\end{array}$} & 2017 & 10 & Sheep & 10 & 2 & PMMA & Control & Femur & 12 & $\begin{array}{l}500 \mu \mathrm{m} \text { micro- } \\
\text { motion }\end{array}$ & $\begin{array}{l}\text { Every gait } \\
\text { cycle }\end{array}$ & & 500 & & \\
\hline & & & & & & & Zoledronate & & 12 & $\begin{array}{l}500 \mu \mathrm{m} \text { micro- } \\
\text { motion }\end{array}$ & $\begin{array}{l}\text { Every gait } \\
\text { cycle }\end{array}$ & & 500 & & \\
\hline Jasty & 1997 & 20 & Dogs & 5 & 4 & Titanium & None & Femur & 6 & Unloaded & N/A & Unloaded & & $9.3 \pm 2.3$ & \\
\hline & & & & & & & & & 6 & $\begin{array}{l}20 \mu \mathrm{m} \text { micro- } \\
\text { motion }\end{array}$ & $8 \mathrm{~h} /$ day & 20 & & $9.0 \pm 3.1$ & \\
\hline & & & & & & & & & 6 & $\begin{array}{l}40 \mu \mathrm{m} \text { micro- } \\
\text { motion }\end{array}$ & $8 \mathrm{~h} /$ day & & 40 & $11.8 \pm 3.9$ & \\
\hline & & & & & & & & & 6 & $\begin{array}{l}150 \mu \mathrm{m} \text { micro- } \\
\text { motion }\end{array}$ & $8 \mathrm{~h} /$ day & & 150 & $10.4 \pm 3.0$ & \\
\hline Kawahara & 2003 & & Beagles & & & Titanium & None & $\begin{array}{l}\text { Mandi- } \\
\text { ble }\end{array}$ & 6 & $8 \mathrm{~N}$ & $10 \mathrm{~s}$ & 30 & 580,630 & & not measured \\
\hline $\begin{array}{l}\text { Over- } \\
\text { gaard }\end{array}$ & 1996 & 14 & Dogs & 7 & 2 & Titanium & $\begin{array}{l}\text { Hydroxyapa- } \\
\text { tite coated }\end{array}$ & Femur & 16 & \begin{tabular}{|l|}
$150 \mu \mathrm{m}$ \\
micromotion \\
$(4$ weeks), \\
then unloaded \\
(12 weeks)
\end{tabular} & Everyday & 150 & & $28.5 \pm 8.8$ & $54.6 \pm 10.0$ \\
\hline & & & & & & & & & 16 & $\begin{array}{l}150 \mu \mathrm{m} \text { micro- } \\
\text { motion }\end{array}$ & Everyday & & 150 & $24.1 \pm 16.1$ & $37.7 \pm 10.1$ \\
\hline Soballe & 1992 & 14 & Dogs & 8 & 4 & Titanium & $\begin{array}{l}\text { Hydroxyapa- } \\
\text { tite coated }\end{array}$ & Femur & 4 & $\begin{array}{l}500 \mu \mathrm{m} \text { micro- } \\
\text { motion }\end{array}$ & $\begin{array}{l}\text { every gait } \\
\text { cycle }\end{array}$ & & 500 & & $0-10 \%$ \\
\hline & & & & & & & $\begin{array}{l}\text { Hydroxyapa- } \\
\text { tite coated }\end{array}$ & & 4 & Unloaded & N/A & unloaded & & & $45 \%$ \\
\hline & & & & & & & \begin{tabular}{|l|}
$\begin{array}{l}\text { Titanium } \\
\text { coated }\end{array}$ \\
\end{tabular} & & 4 & $\begin{array}{l}500 \mu \mathrm{m} \text { micro- } \\
\text { motion }\end{array}$ & $\begin{array}{l}\text { every gait } \\
\text { cycle }\end{array}$ & & 500 & & $0-10 \%$ \\
\hline
\end{tabular}




\begin{tabular}{|c|c|c|c|c|c|c|c|c|c|c|c|c|c|c|c|}
\hline \multirow[b]{2}{*}{ Author } & \multirow[b]{2}{*}{ Year } & \multirow[b]{2}{*}{$\begin{array}{l}\text { No. of } \\
\text { animals or } \\
\text { patients }\end{array}$} & \multirow[b]{2}{*}{ Species } & \multirow{2}{*}{$\begin{array}{l}\text { No. of } \\
\text { samples } \\
\text { (per } \\
\text { group) }\end{array}$} & \multirow[b]{2}{*}{$\begin{array}{l}\text { No. of } \\
\text { study } \\
\text { groups }\end{array}$} & \multirow[b]{2}{*}{$\begin{array}{l}\text { Implant } \\
\text { material }\end{array}$} & \multirow[b]{2}{*}{$\begin{array}{l}\text { Implant } \\
\text { coating or } \\
\text { implant type }\end{array}$} & \multirow[b]{2}{*}{ Bone } & \multirow[b]{2}{*}{\begin{tabular}{|l} 
Time \\
(weeks)
\end{tabular}} & \multirow[b]{2}{*}{$\begin{array}{l}\text { Loading } \\
\text { conditions }\end{array}$} & \multirow[b]{2}{*}{$\begin{array}{l}\text { Loading } \\
\text { cycles and } \\
\text { time }\end{array}$} & \multicolumn{2}{|c|}{ Micromotion $(\mu \mathrm{m})$} & \multirow[b]{2}{*}{$\begin{array}{l}\text { Bone } \\
\text { ingrowth }\end{array}$} & \multirow[b]{2}{*}{$\begin{array}{l}\text { Bone- } \\
\text { implant- } \\
\text { contact }\end{array}$} \\
\hline & & & & & & & & & & & & $\begin{array}{l}\text { Osseo- } \\
\text { integrated }\end{array}$ & $\begin{array}{l}\text { Non-osseo- } \\
\text { integrated }\end{array}$ & & \\
\hline & & & & & & & $\begin{array}{l}\text { Titanium } \\
\text { coated }\end{array}$ & & 4 & Unloaded & N/A & Unloaded & & & $0-10 \%$ \\
\hline \multirow[t]{3}{*}{ Soballe } & 1992 & 14 & Dogs & 7 & 4 & Titanium & $\begin{array}{l}\text { Hydroxyapa- } \\
\text { tite coated }\end{array}$ & Femur & 4 & $\begin{array}{l}150 \mu \mathrm{m} \text { micro- } \\
\text { motion }\end{array}$ & $\begin{array}{l}\text { Every gait } \\
\text { cycle }\end{array}$ & 150 & & & $7 \pm 2$ \\
\hline & & & & & & & $\begin{array}{l}\text { Hydroxyapa- } \\
\text { tite coated }\end{array}$ & & 4 & unloaded & N/A & unloaded & & & $65 \pm 2$ \\
\hline & & & & & & & $\begin{array}{l}\text { Titanium } \\
\text { coated }\end{array}$ & & 4 & $\begin{array}{l}150 \mu \mathrm{m} \text { micro- } \\
\text { motion }\end{array}$ & $\begin{array}{l}\text { Every gait } \\
\text { cycle }\end{array}$ & & 150 & & 0 \\
\hline \multirow[t]{3}{*}{$\begin{array}{l}\text { Van- } \\
\text { damme a }\end{array}$} & 2007 & 14 & Rabbits & 10 & 3 & Titanium & None & Tibia & 12 & Unloaded & N/A & & unloaded & & $0-20 \%$ \\
\hline & & & & 10 & & & & & 6 & $\begin{array}{l}30 \mu \mathrm{m} \text { micro- } \\
\text { motion }\end{array}$ & $\begin{array}{l}400 \text { cycles/ } \\
\text { day; twice/ } \\
\text { week }\end{array}$ & & 30 & & $0-20 \%$ \\
\hline & & & & 11 & & & & & 12 & \begin{tabular}{|l}
$30 \mu \mathrm{m}$ \\
micromotion \\
$(6$ weeks), \\
then $50 \mu \mathrm{m}$ \\
micromotion \\
( 6 weeks)
\end{tabular} & $\begin{array}{l}400 \text { cycles/ } \\
\text { day; twice/ } \\
\text { week, then } \\
800 \text { cycles/ } \\
\text { day; twice/ } \\
\text { week }\end{array}$ & 30,50 & & & $60-80 \%$ \\
\hline \multirow[t]{3}{*}{$\begin{array}{l}\text { Van- } \\
\text { damme b }\end{array}$} & 2007 & 10 & rabbits & 10 & 3 & Titanium & none & Tibia & 9 & Unloaded & & unloaded & & & 42.22 \\
\hline & & & & & & & & & 9 & $\begin{array}{l}30 \mu \mathrm{m} \text { micro- } \\
\text { motion }\end{array}$ & $\begin{array}{l}400 \text { cycles/ } \\
\text { day; thrice/ } \\
\text { week }\end{array}$ & 30 & & & 71.43 \\
\hline & & & & & & & & & 9 & $\begin{array}{l}90 \mu \mathrm{m} \text { micro- } \\
\text { motion }\end{array}$ & $\begin{array}{l}400 \text { cycles/ } \\
\text { day; thrice/ } \\
\text { week }\end{array}$ & 90 & & & 74.36 \\
\hline \multirow[t]{4}{*}{$\begin{array}{l}\text { Van- } \\
\text { damme c }\end{array}$} & 2008 & 20 & Rabbits & 10 & 2 & Titanium & Turned & Tibia & 9 & Unloaded & N/A & unloaded & & & 6.98 \\
\hline & & & & & & & Turned & & 9 & $\begin{array}{l}30 \mu \mathrm{m} \text { micro- } \\
\text { motion }\end{array}$ & $\begin{array}{l}400 \text { cycles/ } \\
\text { day; thrice/ } \\
\text { week }\end{array}$ & 30 & & & 53.33 \\
\hline & & & & & & & Roughened & & 9 & Unloaded & N/A & Unloaded & & & 42.22 \\
\hline & & & & & & & Roughened & & 9 & $\begin{array}{l}30 \mu \mathrm{m} \text { micro- } \\
\text { motion }\end{array}$ & $\begin{array}{l}400 \text { cycles/ } \\
\text { day; thrice/ } \\
\text { week }\end{array}$ & 30 & & & 71.43 \\
\hline \multirow[t]{4}{*}{$\begin{array}{l}\text { Van- } \\
\text { damme d }\end{array}$} & 2007 & 10 & Rabbits & 10 & 3 & Titanium & screw & Tibia & 9 & Unloaded & N/A & & unloaded & & $0-3 \%$ \\
\hline & & & & & & & screw & & 9 & $\begin{array}{l}30 \mu \mathrm{m} \text { micro- } \\
\text { motion }\end{array}$ & $\begin{array}{l}400 \text { cycles/ } \\
\text { day; thrice/ } \\
\text { week }\end{array}$ & 30 & & & $9-20 \%$ \\
\hline & & & & & & & cylindrical & & 9 & $\begin{array}{l}30 \mu \mathrm{m} \text { micro- } \\
\text { motion }\end{array}$ & $\begin{array}{l}400 \text { cycles/ } \\
\text { day; thrice/ } \\
\text { week }\end{array}$ & 30 & & & $0-8 \%$ \\
\hline & & & & & & & titanium & & 4 & Unloaded & N/A & Unloaded & & & $13 \pm 3$ \\
\hline \multirow[t]{2}{*}{ Manley } & 1995 & 12 & Dogs & 6 & 2 & Titanium & Collared & Femur & 16 & $\pm 50 \mathrm{~N}$ & $\begin{array}{l}16 \mathrm{~s} \text { at } \\
0.5 \mathrm{~Hz}\end{array}$ & $33 \pm 23.7$ & & & $52 \pm 11.4$ \\
\hline & & & & & & & Collarless & & 16 & $\pm 50 \mathrm{~N}$ & $\begin{array}{l}16 \mathrm{~s} \text { at } \\
0.5 \mathrm{~Hz}\end{array}$ & $17 \pm 4.2$ & & & $42 \pm 8.5$ \\
\hline Pilliar & 1986 & & Dogs & 5 & 3 & $\begin{array}{l}\text { Cobalt } \\
\text { Chrome }\end{array}$ & & Femur & 52 & & & 28 & 150 & & \\
\hline \multirow[t]{2}{*}{ Trisi a } & 2017 & 2 & sheep & 10 & 2 & Titanium & SLA & Iliac crest & 8 & $25 \mathrm{~N} / \mathrm{mm}$ & \begin{tabular}{|l}
$\begin{array}{l}\text { End point } \\
\text { analysis }\end{array}$ \\
\end{tabular} & $77.9 \pm 17.29$ & & & $49.49 \pm 7.70$ \\
\hline & & & & & & & FEL & & 8 & $25 \mathrm{~N} / \mathrm{mm}$ & $\begin{array}{l}\begin{array}{l}\text { End point } \\
\text { analysis }\end{array} \\
\end{array}$ & $75.3 \pm 19$ & & & $65.33 \pm 6.35$ \\
\hline \multirow[t]{4}{*}{ Trisi b } & 2015 & 4 & Sheep & 20 & 2 & Titanium & Large threaded & Iliac crest & 8 & $25 \mathrm{~N} / \mathrm{mm}$ & $\begin{array}{l}\text { End point } \\
\text { analysis }\end{array}$ & $64 \pm 27$ & & & $50.58 \pm 8.65$ \\
\hline & & & & & & & small threaded & & 8 & $25 \mathrm{~N} / \mathrm{mm}$ & $\begin{array}{l}\text { End point } \\
\text { analysis }\end{array}$ & & $177 \pm 87$ & & $40.98 \pm 14.03$ \\
\hline & & & & & & & Large threaded & $\begin{array}{l}\text { Mandi- } \\
\text { ble }\end{array}$ & 8 & $25 \mathrm{~N} / \mathrm{mm}$ & $\begin{array}{l}\text { End point } \\
\text { analysis }\end{array}$ & $15 \pm 5$ & & & $36.1 \pm 18.3$ \\
\hline & & & & & & & small threaded & & 8 & $25 \mathrm{~N} / \mathrm{mm}$ & \begin{tabular}{|l|}
$\begin{array}{l}\text { End point } \\
\text { analysis }\end{array}$ \\
\end{tabular} & $22 \pm 6$ & & & $34.06 \pm 18.18$ \\
\hline \multirow[t]{2}{*}{ Trisi c } & 2016 & 2 & Sheep & 10 & 2 & Titanium & $\begin{array}{l}\text { Coventional } \\
\text { drill }\end{array}$ & Iliac crest & 8 & $25 \mathrm{~N} / \mathrm{mm}$ & $\begin{array}{l}\begin{array}{l}\text { End point } \\
\text { analysis }\end{array} \\
\end{array}$ & $94.88 \pm 10.94$ & & & $46.19 \pm 3.98$ \\
\hline & & & & & & & $\begin{array}{l}\text { Osseo-densifi- } \\
\text { cation }\end{array}$ & & 8 & $25 \mathrm{~N} / \mathrm{mm}$ & $\begin{array}{l}\text { End point } \\
\text { analysis }\end{array}$ & $60.45 \pm 5.29$ & & & $49.58 \pm 3.19$ \\
\hline Trisi d & 2016 & 2 & Sheep & 24 & 2 & Titanium & Healthy & Iliac crest & 8 & $25 \mathrm{~N} / \mathrm{cm}$ & $\begin{array}{l}\text { End point } \\
\text { analysis }\end{array}$ & $161.26 \pm 134.39$ & & & $44.75 \pm 9.77$ \\
\hline & & & & & & & Failed & & 8 & $25 \mathrm{~N} / \mathrm{cm}$ & $\begin{array}{l}\text { End point } \\
\text { analysis }\end{array}$ & & $619.5 \pm 328.26$ & & $22.6 \pm 9.54$ \\
\hline Engh & 1992 & $\begin{array}{l}14(6 \\
\text { female) }\end{array}$ & $\begin{array}{l}\text { Human, } \\
\text { mean } \\
\text { age } 71\end{array}$ & 14 & 1 & $\begin{array}{l}\text { Cobalt } \\
\text { Chrome }\end{array}$ & $\begin{array}{l}\text { Coated hip } \\
\text { stem }\end{array}$ & Femur & $52-403$ & $\begin{array}{l}\text { Gait \& stair } \\
\text { climbing }\end{array}$ & N/A & $<40$ & 150 & & \\
\hline
\end{tabular}

Table 2. Detailed study characteristics of the selected studies. 


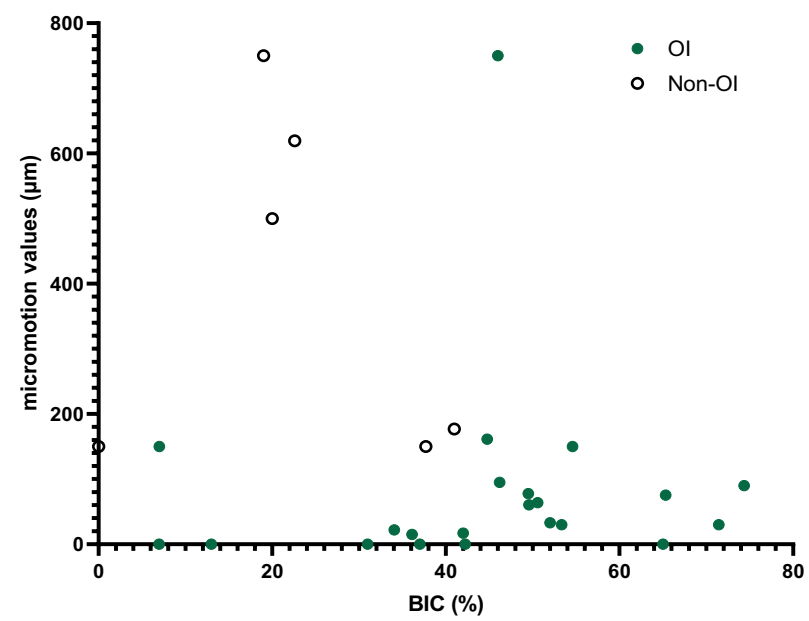

Figure 4. Scatter plot graph of the correlation between micromotion values and percentage BIC for the animal studies. For the osseointegrated data (green filled circles) a positive correlation was found (Spearman's $\rho=0.41, p$ value $=0.02$ ). No correlation was observed for the non-osseointegrated data (empty circles), nor the full dataset (all circles).

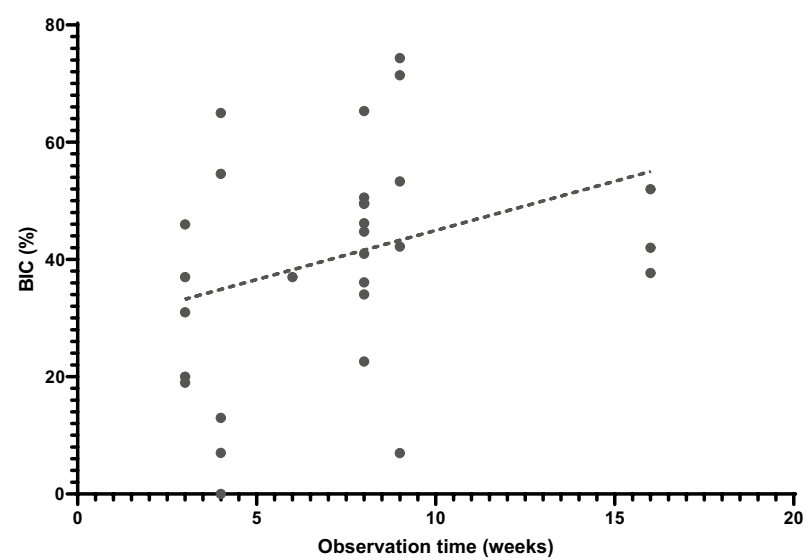

Figure 5. Scatter plot demonstrating the correlation between observation time and percentage BIC for the animal studies. Spearman's $\rho=0.40, p$ value $=0.01$.

a cumulative meta-analysis to quantify the risk of bias between studies. Rather, to provide some quantitative analysis we applied Mann Whitney tests to the data extracted from each study. Further we isolated the effects of studies that applied micromotion, and those that measured it, and found that this did not affect the principal finding our systematic review (Fig. 3).

In conclusion, this systematic review has demonstrated that the idea of a universal limit of tolerable micromotion for implant osseointegration is misleading. Rather, implant and environmental factors, and their link to interfacial stress-strain states, must be considered to identify the most appropriate limit for the biomaterial/ patient group under consideration. The tables provided in this systematic review summarise the implant and environmental conditions for all published quantitative in vivo micromotion research and will enable investigators to compare their data to the most appropriate values.

\section{Materials and methods}

Protocol and registration. Prior to the investigation, the protocol was registered with the International prospective register for systematic reviews PROSPERO (ID: CRD42020196686), following the Preferred Reporting Items for Systematic Reviews and Meta-Analyses (PRISMA) statement and checklist ${ }^{68}$.

Eligibility criteria. Studies which fulfilled the following criteria were included: (1) in vivo animal research or post-mortem human data where the implant was inserted pre-mortem. (2) testing of osseointegration when micromotion was either applied or measured with micromotion values reported in the form of displacement (3) the study was an original research article; and (4) the studies were published in English.

Articles were excluded if: (1) micromotion was measured indirectly and/or reported as implant stability quotient (ISQ) or resonance frequency analysis (RFA); (2) Study duration less than 3 weeks; (3) Finite element 
analysis (FEA) or computational studies; (4) cemented implants; (5) cadaveric bone in vitro experiments where prostheses were inserted post-mortem; and (6) synthetic bone in vitro experiments.

Information sources and search strategy. An electronic search was performed for articles published up to $16^{\text {th }}$ November 2020, in the following databases: PubMed, Scopus and Web of Science. The search strategy identified papers which included the following terms: (micromotion OR "micro-motion" OR "micro motion") AND ("osseointegration" OR "osteointegration").

Study selection. Two independent reviewers (N.K., J.S.) assessed the titles and abstracts of all the studies and discarded studies that met any of the exclusion criteria. The full text of all remaining studies was then assessed against the inclusion and exclusion criteria. Any disagreement regarding eligibility of articles were resolved by a third reviewer (R.v.A.).

Data collection process and data items. Data relating to osseointegrated and non-osseointegrated values of micromotion were extracted. The country, animal species, number of study groups, duration of the experiment, implant material and loading conditions were recorded. The outcome of osseointegration measured as bony ingrowth or percentage bone-implant-contact (BIC) were also recorded.

The micromotion methodology (applied or measured) was also recorded. In the applied group, known values of micromotion in the form of cyclic loading were directly applied as a controlled experimental condition and then osseointegration was assessed. In measured group, micromotion was not a controlled experimental condition, rather micromotion at the bone-implant interface was measured once the implant had osseointegrated/not.

Statistical analysis. Data were analysed and plotted using Graph Pad Prism 8 software and have been reported as mean \pm standard deviation $(\mathrm{SD})$. Four analyses were performed:

(1) All micromotion values were grouped into osseointegrated/not, according to the definition used by the original study authors. Data were first tested for normality, and then non-parametric Mann Whitney tests were used to compare differences between groups.

(2) Micromotion values were further discretised according to the study method (applied vs measured micromotion). Then analysis 1) was repeated for both of these subgroups.

(3) Spearman correlation tests were used to examine correlation between percentage BIC and micromotion values for three groups: all data, osseointegrated, non-osseointegrated.

(4) Spearman correlation tests were used to examine the correlation between percentage BIC and study duration.

The significance level was set to $\alpha=0.05$.

\section{Data availability}

Data generated and analysed during this study are included in this published article. Data are available from the corresponding author subject to reasonable request.

Received: 7 January 2021; Accepted: 4 May 2021

Published online: 24 May 2021

\section{References}

1. Pabinger, C., Lothaller, H. \& Geissler, A. Utilization rates of knee-arthroplasty in OECD countries. Osteoarthritis Cartilage 23, 1664-1673. https://doi.org/10.1016/j.joca.2015.05.008 (2015).

2. Irandoust, S. \& Müftü, S. The interplay between bone healing and remodeling around dental implants. Sci. Rep. 10, 1-10 (2020).

3. Barnes, S. C., Clasper, J. C., Bull, A. M. \& Jeffers, J. R. Micromotion and push-out evaluation of an additive manufactured implant for above-the-knee amputees. J. Orthop. Res. 37, 2104-2111 (2019).

4. Trisi, P. et al. Implant micromotion is related to peak insertion torque and bone density. Clin. Oral. Implant Res. 20, 467-471 (2009).

5. Han, S. et al. Micromotion and migration of cementless tibial trays under functional loading conditions. J. Arthroplasty 36, 349-355. https://doi.org/10.1016/j.arth.2020.07.017 (2021).

6. Haïat, G., Wang, H.-L. \& Brunski, J. Effects of biomechanical properties of the bone-implant interface on dental implant stability: from in silico approaches to the patient's mouth. Annu. Rev. Biomed. Eng. 16, 187-213. https://doi.org/10.1146/annurev-bioeng071813-104854 (2014).

7. Winter, W., Klein, D. \& Karl, M. Micromotion of dental implants: basic mechanical considerations. J. Med. Eng. 265412-265412, 2013. https://doi.org/10.1155/2013/265412 (2013).

8. Trisi, P., Berardini, M., Falco, A. \& Vulpiani, M. P. Effect of implant thread geometry on secondary stability, bone density, and bone-to-implant contact: a biomechanical and histological analysis. Implant Dent. 24, 384-391 (2015).

9. Ellenrieder, M., Souffrant, R., Schulze, C., Mittelmeier, W. \& Bader, R. Micromotion and subsidence of a cementless conical fluted stem depending on femoral defect size - A human cadaveric study. Clin. Biomech. 80, 105202. https://doi.org/10.1016/j.clinb iomech.2020.105202 (2020).

10. Apostu, D., Lucaciu, O., Berce, C., Lucaciu, D. \& Cosma, D. Current methods of preventing aseptic loosening and improving osseointegration of titanium implants in cementless total hip arthroplasty: a review. J. Int. Med. Res. 46, 2104-2119 (2018).

11. Shah, F. A., Thomsen, P. \& Palmquist, A. Osseointegration and current interpretations of the bone-implant interface. Acta Biomater. 84, 1-15 (2019).

12. Piuzzi, N. S. Patient-reported outcome measures (pain, function, and quality of life) after aseptic revision total knee arthroplasty. J. Bone Joint Surg. Am. 102, e114 (2020).

13. Raffa, M. L., Nguyen, V.-H. \& Haiat, G. Micromechanical modeling of the contact stiffness of an osseointegrated bone-implant interface. Biomed. Eng. Online 18, 1-18 (2019). 
14. Galbusera, F. et al. Biomechanics of sacropelvic fixation: a comprehensive finite element comparison of three techniques. Eur. Spine J. 29, 295-305 (2020).

15. Xie, S. et al. A finite element analysis of tibial tritanium cones without stems in varying bone defects. Knee 27, 656-666 (2020).

16. Chanda, S., Mukherjee, K., Gupta, S. \& Pratihar, D. K. A comparative assessment of two designs of hip stem using rule-based simulation of combined osseointegration and remodelling. Proc. Inst. Mech. Eng. H 234, 118-128 (2020).

17. de Barros e Lima Bueno, R., Dias, A. P., Ponce, K. J., Brunski, J. B. \& Nanci, A. System for application of controlled forces on dental implants in rat maxillae: influence of the number of load cycles on bone healing. J. Biomed. Mater. Res. B 108, 965-975 (2020).

18. Trisi, P., Falco, A. \& Berardini, M. Single-drill implant induces bone corticalization during submerged healing: an in vivo pilot study. Int. J. Implant Dentistry 6, 1-6 (2020).

19. Chen, J., Xiao, Z., Yangpeng, S., Deng, F. \& Zhiguang, Z. Production of inter-connective porous dental implants by computer-aided design and metal three-dimensional printing. J. Biomater. Appl. 34, 1227-1238 (2020).

20. Floerkemeier, T. et al. Greater early migration of a short-stem total hip arthroplasty is not associated with an increased risk of osseointegration failure: 5th-year results from a prospective RSA study with 39 patients, a follow-up study. Acta Orthop. 91, 266-271 (2020).

21. Jørgensen, P. B. et al. Higher early proximal migration of hemispherical cups with electrochemically applied hydroxyapatite (BoneMaster) on a porous surface compared with porous surface alone: A randomized RSA study with 53 patients. Acta Orthop. 91, 26-32 (2020).

22. O'Rourke, D. \& Taylor, M. Patient and surgical variability in the primary stability of cementless acetabular cups: a finite element study. J. Orthop. Res. 38, 1515-1522 (2020).

23. Søballe, K., Mouzin, O. R., Kidder, L. A., Overgaard, S. \& Bechtold, J. E. The effects of hydroxyapatite coating and bone allograft on fixation of loaded experimental primary and revision implants. Acta Orthop. Scand. 74, 239-247 (2003).

24. Leucht, P. et al. Effect of mechanical stimuli on skeletal regeneration around implants. Bone 40, 919-930 (2007).

25. Manley, P. A., Vanderby, R. Jr., Kohles, S., Markel, M. D. \& Heiner, J. P. Alterations in femoral strain, micromotion, cortical geometry, cortical porosity, and bony ingrowth in uncemented collared and collarless prostheses in the dog. J. Arthroplasty 10, 63-73 (1995).

26. Ovesy, M., Voumard, B. \& Zysset, P. A nonlinear homogenized finite element analysis of the primary stability of the bone-implant interface. Biomech. Model. Mechanobiol. 17, 1471-1480 (2018).

27. Ghoul, W. E. \& Chidiac, J. J. Prosthetic requirements for immediate implant loading: a review. J. Prosthodontics Implant Esthetic Reconstruct. Dentistry 21, 141-154 (2012).

28. van Arkel, R. J., Ghouse, S., Milner, P. E. \& Jeffers, J. R. T. Additive manufactured push-fit implant fixation with screw-strength pull out. J. Orthop. Res. 36, 1508-1518. https://doi.org/10.1002/jor.23771 (2018).

29. Kawahara, H. et al. Osseointegration under immediate loading: biomechanical stress-strain and bone formation-resorption. Implant Dent. 12, 61-68 (2003).

30. Vandamme, K. et al. Histodynamics of bone tissue formation around immediately loaded cylindrical implants in the rabbit. Clin. Oral. Implant Res. 18, 471-480 (2007).

31. Goodman, S., Wang, J. S., Doshi, A. \& Aspenberg, P. Difference in bone ingrowth after one versus two daily episodes of micromotion: experiments with titanium chambers in rabbits. J. Biomed. Mater. Res. 27, 1419-1424 (1993).

32. Aspenberg, P., Goodman, S., Toksvig-Larsen, S., Ryd, L. \& Albrektsson, T. Intermittent micromotion inhibits bone ingrowth: titanium implants in rabbits. Acta Orthop. Scand. 63, 141-145 (1992).

33. Bragdon, C. R. et al. Differences in stiffness of the interface between a cementless porous implant and cancellous bone in vivo in dogs due to varying amounts of implant motion. J. Arthroplasty 11, 945-951. https://doi.org/10.1016/S0883-5403(96)80136-7 (1996).

34. Duyck, J. et al. The influence of micro-motion on the tissue differentiation around immediately loaded cylindrical turned titanium implants. Arch. Oral. Biol. 51, 1-9. https://doi.org/10.1016/j.archoralbio.2005.04.003 (2006).

35. Goodman, S. B., Aspenberg, P., Song, Y., Regula, D. \& Lidgren, L. Intermittent micromotion and polyethylene particles inhibit bone ingrowth into titanium chambers in rabbits. J. Appl. Biomater. 6, 161-165 (1995).

36. Goodman, S. B., Song, Y., Doshi, A. \& Aspenberg, P. Cessation of strain facilitates bone formation in the micromotion chamber implanted in the rabbit tibia. Biomaterials 15, 889-893 (1994).

37. Goodman, S., Toksvig-Larsen, S. \& Aspenberg, P. Ingrowth of bone into pores in titanium chambers implanted in rabbits: effect of pore cross-sectional shape in the presence of dynamic shear. J. Biomed. Mater. Res. 27, 247-253 (1993).

38. Jakobsen, T., Kold, S., Baas, J., Søballe, K. \& Rahbek, O. Sheep hip Arthroplasty model of failed implant Osseointegration. Open Orthopaedics J. 9, 525 (2015).

39. Jakobsen, T. et al. Topical zoledronic acid decreases micromotion induced bone resorption in a sheep arthroplasty model. BMC Musculoskel Dis. 18, 1-7 (2017).

40. Jasty, M. et al. In vivo skeletal responses to porous-surfaced implants subjected to small induced motions. J. Bone Joint Surg. Am. 79, 707-714 (1997).

41. Overgaard, S., Søballe, K., Josephsen, K., Hansen, E. \& Bünger, C. Role of different loading conditions on resorption of hydroxyapatite coating evaluated by histomorphometric and stereological methods. J. Orthop. Res. 14, 888-894 (1996).

42. Søballe, K., Hansen, E. S., Rasmussen, B. H., Jørgensen, P. H. \& Bünger, C. Tissue ingrowth into titanium and hydroxyapatite-coated implants during stable and unstable mechanical conditions. J. Orthop. Res. 10, 285-299. https://doi.org/10.1002/jor.1100100216 (1992).

43. Søballe, K., Brockstedt-Rasmussen, H., Hansen, E. S. \& Bünger, C. Hydroxyapatite coating modifies implant membrane formation: controlled micromotion studied in dogs. Acta Orthop. Scand. 63, 128-140 (1992).

44. Vandamme, K. et al. The effect of micro-motion on the tissue response around immediately loaded roughened titanium implants in the rabbit. Eur. J. Oral Sci. 115, 21-29. https://doi.org/10.1111/j.1600-0722.2007.00416.x (2007).

45. Vandamme, K., Naert, I., Vander Sloten, J., Puers, R. \& Duyck, J. Effect of implant surface roughness and loading on peri-implant bone formation. J. Periodontol. 79, 150-157 (2008).

46. Vandamme, K. et al. Influence of controlled immediate loading and implant design on peri-implant bone formation. J. Clin. Periodontol. 34, 172-181 (2007).

47. Pilliar, R. M., Lee, J. M. \& Maniatopoulos, C. Observations on the effect of movement on bone ingrowth into porous-surfaced implants. Clin Orthop Relat Res, 108-113 (1986).

48. Trisi, P., Berardini, M., Falco, A., Sandrini, E. \& Vulpiani, M. P. A new highly hydrophilic electrochemical implant titanium surface: a histological and biomechanical Vivo: Study. Implant Dent. 26, 429-437 (2017).

49. Trisi, P., Berardini, M., Falco, A. \& Podaliri Vulpiani, M. New Osseodensification implant site preparation method to increase bone density in low-density bone: in vivo evaluation in sheep. Implant Dent. 25, 24-31. https://doi.org/10.1097/ID.0000000000000358 (2016).

50. Trisi, P., Berardini, M., Falco, A. \& Podaliri Vulpiani, M. Validation of value of actual micromotion as a direct measure of implant micromobility after healing (secondary implant stability). An in vivo histologic and biomechanical study. Clin. Oral Implants Res. 27, 1423-1430. https://doi.org/10.1111/clr.12756 (2016).

51. Engh, C. A. et al. Quantification of implant micromotion, strain shielding, and bone resorption with porous-coated anatomic medullary locking femoral prostheses. Clin Orthop Relat R, 13-29 (1992). 
52. Breding, K. et al. The effect of hydroxyapatite nanocrystals on osseointegration of titanium implants: an $<\mathrm{i}>$ In Vivo $</ \mathrm{i}>$ rabbit study. Int. J. Dentistry https://doi.org/10.1155/2014/171305 (2014).

53. Meirelles, L. et al. Nano hydroxyapatite structures influence early bone formation. J. Biomed. Mater. Res., Part A 87A, 299-307. https://doi.org/10.1002/jbm.a.31744 (2008).

54. Robling, A. G., Burr, D. B. \& Turner, C. H. Recovery periods restore mechanosensitivity to dynamically loaded bone. J. Exp. Biol. 204, 3389 (2001).

55. Saxon, L. K., Robling, A. G., Alam, I. \& Turner, C. H. Mechanosensitivity of the rat skeleton decreases after a long period of loading, but is improved with time off. Bone 36, 454-464. https://doi.org/10.1016/j.bone.2004.12.001 (2005).

56. Parithimarkalaignan, S. \& Padmanabhan, T. Osseointegration: an update. J. Indian Prosthodontic Soc. 13, 2-6 (2013).

57. Kohli, N. et al. Bone remodelling in vitro: Where are we headed?:-A review on the current understanding of physiological bone remodelling and inflammation and the strategies for testing biomaterials in vitro. Bone 110, 38-46 (2018).

58. Wazen, R. M. et al. Micromotion-induced strain fields influence early stages of repair at bone-implant interfaces. Acta Biomater 9, 6663-6674. https://doi.org/10.1016/j.actbio.2013.01.014 (2013).

59. Simmons, C. A., Meguid, S. A. \& Pilliar, R. M. Mechanical regulation of localized and appositional bone formation around boneinterfacing implants. J. Biomed. Mater. Res. 55, 63-71. https://doi.org/10.1002/1097-4636(200104)55:1\%3c63::AID-JBM90\%3e3.0. $\mathrm{CO} ; 2-\mathrm{V}(2001)$.

60. Raffa, M. L., Nguyen, V.-H., Hernigou, P., Flouzat-Lachaniette, C.-H. \& Haiat, G. Stress shielding at the bone-implant interface: Influence of surface roughness and of the bone-implant contact ratio. J. Orthop. Res. https://doi.org/10.1002/jor.24840 (2020)

61. Simmons, C. A., Meguid, S. A. \& Pilliar, R. M. Differences in osseointegration rate due to implant surface geometry can be explained by local tissue strains. J. Orthop. Res. 19, 187-194 (2001).

62. Claes, L. E. \& Heigele, C. A. Magnitudes of local stress and strain along bony surfaces predict the course and type of fracture healing. J. Biomech. 32, 255-266. https://doi.org/10.1016/S0021-9290(98)00153-5 (1999).

63. Carter, D. R., Beaupré, G. S., Giori, N. J. \& Helms, J. A. Mechanobiology of skeletal regeneration. Clin. Orthop. Relat. Res. 19762007(355), S41-S55 (1998).

64. Perren, S. M. Evolution of the internal fixation of long bone fractures: the scientific basis of biological internal fixation: choosing a new balance between stability and biology. J. Bone Joint Surg. Br. 84, 1093-1110 (2002).

65. Geris, L., Vander Sloten, J. \& Van Oosterwyck, H. In silico biology of bone modelling and remodelling: regeneration. Philosophical Trans. Royal Soc. A Math. Phys. Eng. Sci. 367, 2031-2053 (2009).

66. Amor, N., Geris, L., Vander Sloten, J. \& Van Oosterwyck, H. Modelling the early phases of bone regeneration around an endosseous oral implant. Comput. Methods Biomed. 12, 459-468 (2009).

67. Borenstein, M., Hedges, L. V., Higgins, J. P. T. \& Rothstein, H. R. Introduction Meta Anal. https://doi.org/10.1002/9780470743386 (2009).

68. Page, M. J. et al. The PRISMA 2020 statement: an updated guideline for reporting systematic reviews. BMJ 372, n71. https://doi. org/10.1136/bmj.n71 (2021).

\section{Acknowledgements}

This work was supported by UKRI (EPSRC) EP/S022546/1 (N.K. and R.v.A.) and the Peter Stormonth Darling Charitable Trust (J.S.).

\section{Author contributions}

N.K. and R.v.A. designed the study. N.K. and J.S. collected and analysed the data. R.v.A. acted as a third reviewed to resolve issues regarding selection of articles for data synthesis. N.K. prepared all the figures and tables. N.K. and R.v.A. wrote the final manuscript. All authors reviewed the manuscript.

\section{Competing interests}

The authors declare no competing interests.

\section{Additional information}

Correspondence and requests for materials should be addressed to R.J.v.A.

Reprints and permissions information is available at www.nature.com/reprints.

Publisher's note Springer Nature remains neutral with regard to jurisdictional claims in published maps and institutional affiliations.

Open Access This article is licensed under a Creative Commons Attribution 4.0 International License, which permits use, sharing, adaptation, distribution and reproduction in any medium or format, as long as you give appropriate credit to the original author(s) and the source, provide a link to the Creative Commons licence, and indicate if changes were made. The images or other third party material in this article are included in the article's Creative Commons licence, unless indicated otherwise in a credit line to the material. If material is not included in the article's Creative Commons licence and your intended use is not permitted by statutory regulation or exceeds the permitted use, you will need to obtain permission directly from the copyright holder. To view a copy of this licence, visit http://creativecommons.org/licenses/by/4.0/.

(C) The Author(s) 2021 